
\title{
25 Research Soure \\ QTL mapping for root traits in wheat under different phosphorus levels in hydroponic culture
}

\author{
Mengjiao Yang \\ Institute of Crop Sceience,CAAS \\ Cairong Wang \\ Agricultural Research Institue of Yili \\ Muhammad Adeel Hassan \\ Institute of Crop Science,CAAS \\ Faji Li \\ Institute of Crop Science,CAAS \\ Xianchun Xia \\ Institute of Crop Science,CAAS \\ Shubing Shi \\ College of Agronomy,Xinjiang Agricultural University \\ Yonggui Xiao ( $\nabla$ xiaoyonggui@caas.cn ) \\ Institute of Crop Science,CAAS https://orcid.org/0000-0002-9011-222X \\ Zhonghu He \\ Institute of Crop Science,CAAS
}

\section{Research article}

Keywords: Genome-wide linkage mapping, Quantitative trait loci, Root traits, SNP array

Posted Date: August 23rd, 2019

DOI: https://doi.org/10.21203/rs.2.13411/v1

License: (c) (i) This work is licensed under a Creative Commons Attribution 4.0 International License.

Read Full License

Version of Record: A version of this preprint was published at BMC Genomics on March 11th, 2021. See the published version at https://doi.org/10.1186/s12864-021-07425-4. 


\section{Abstract}

Identification of genes for tolerance against phosphorus $(P)$ deficiency is important for wheat production in P-deficient soils. Nine root system architecture (RSA) and root biomass-related traits (RBT) in 198 double haploid lines derived from cross between Yangmai 16/Zhongmai 895 were characterized under zero (control), low, and high P treatments (KH2PO4 contents: $0,0.005,0.25 \mathrm{mmol} / \mathrm{L}$, respectively) in hydroponic culture, and the DH lines and parents were genotyped using Wheat 660K SNP array. All traits showed significant variations among the $\mathrm{DH}$ lines with high heritabilities (0.76 to 0.91 ) and high correlations ( $r=0.59$ to 0.98 ) with each other. Inclusive composite interval mapping using 10,242 markers identified 34 QTLs for all traits in the three P treatments. Of these, 11 QTLs under low P condition on chromosomes 2BL, 4BS (2 QTLs), 4DS (2), 6BL (2), 7AL (2), and 7BL (2) for root length (RL), root tip number (RTN) and ratio of root to shoot dry weight (RRS) explained $4.6-20.4 \%$ of the phenotypic variances. QRRS.caas-4DS (16.64 - 30.66 Mb) was detected in all three $P$ treatments and accounted for 8.4 to $20.4 \%$ of the phenotypic variances. Seven QTLs clusters (C1 to C7) were mapped on chromosomes $3 \mathrm{DL}, 4 \mathrm{BS}, 4 \mathrm{DS}, 6 \mathrm{BL}, 7 \mathrm{AS}, 7 \mathrm{AL}$ and 7BL, of which five (C3 to $\mathrm{C7}$ ) were detected under low $\mathrm{P}$ condition. Cluster C5 on chromosome 7AS (AX-109955164 - AX-109445593) played the key role in modulating RL, RTN and ROSA, with the favorable allele coming from Zhongmai 895. SNPs closely linked to QTLs and clusters could be used for improving root nutrient-use efficiency traits in future breeding.

\section{Background}

Phosphorus $(P)$ is an important macro-element for ensuring plant morphogenesis, productivity and grain quality [1]. $\mathrm{P}$ as phosphate is immobile in most of the soil types that make its application on the soil surface less beneficial for plants. While efficient uptake of P from deep soil depends on plant's underground organs. $\mathrm{P}$ deficiency causes abnormal physiological and biochemical metabolism during plant growth [2]. In crops, an efficient root system is crucial for P-uptake; increase in root to shoot ratio in most of the elite cultivars helps to access $P$ from deep soil or grow longer root hairs to exploit the spatial characteristics of soil [3]. Therefore, optimization of root-related traits could provide a promising avenue to enhance $P$ uptake and increase grain yield [4].

Significant genetic diversity of root-related traits under different nutrient conditions has been considered very important $[5,6]$. These variations could be exploited to improve nutrients uptake by wheat plants and provide a sustainable solution for developing elite cultivars $[2,7,8]$. In wheat, QTLs have been detected for root traits under different $P$ treatments across the 21 chromosomes $[9,10]$. Despite the many genetic interactions which have been determined for the root biomass and root system architecture traits, but still few loci were reported with major effects $[11,12]$. Nowadays, construction of high-density genetic maps has increased the accuracy of quantitative genomic analysis. These maps can help to identify useful loci for root morphological and P-uptake related traits [13].

However, the characterization of root traits under normal field conditions is difficult; traditional methods such as soil columns and soil cores are time-consuming and laborious for screening of large populations 
$[14,15]$. Artificial systems like sand, germination paper and hydroponic based cultures have been used as proxies for characterization of root traits $[4,16]$. Hydroponic culture with digital imaging has given new insight about different aspects of root development compared with sand culture and germination paper experiments. Moreover, hydroponic technique can be applied for rapid screening of large populations. Several important loci and QTL clusters related to root biomass and root system architecture were reported similar in both hydroponic and field trials conditions $[11,12,17]$. There is not report regarding cloning of QTLs for P-uptake related root traits or P uptake efficiency yet. The 660K SNP array in wheat has greatly improved the density of genetic maps for QTL analysis compared with the earlier 90K Array $[18,19]$. This could increase the chance for identification of true loci for complex traits.

The aims of this study were to evaluate phenotypic diversity of Yangmai 16/Zhongmai 895 derived doubled haploid $(\mathrm{DH})$ population for root system architecture and biomass related traits in different $\mathrm{P}$ treatments at seedling stage using hydroponic culture, and to identify QTLs for root system architecture and root biomass-related traits under low and high P conditions using 660K SNP array based highdensity genetic map.

\section{Methods}

\section{Plant materials}

The panel of $198 \mathrm{DH}$ lines from the cross of Yangmai 16/Zhongmai 895 were evaluated for root-related traits in hydroponic culture. All the materials used in this study were provided in a temperature-controlled greenhouse in National Wheat Improvement Centre, Institute of Crop Sciences, Chinese Academy of Agricultural Sciences from March to April 2016, and authenticated by Associate Professor Yonggui Xiao (Chinese Academy of Agricultural Sciences), and available after approval. The female parental Yangmai 16 is a spring wheat cultivar with drought resistance attributes and cover the largest planting area in the Middle and Lower Yangtze River region. Zhongmai 895 is a facultative cultivar and widely cultivated in southern parts of the Yellow and Huai Valleys. Characterized by high yield potential with drought and heat resistance ability strong root early vigor.

\section{Hydroponic culture and experimental design}

Hoagland's nutrient solution was used [20], and three P levels were kept at zero (control), low and high $\mathrm{P}$ contents $\left(\mathrm{KH}_{2} \mathrm{PO}_{4} 0,0.005,0.25 \mathrm{mmol} / \mathrm{L}\right.$, respectively). $\mathrm{KCl}$ at $0.35,0.345$ and $0.10 \mathrm{mmol} / \mathrm{L}$, respectively, was added in the three treatments to maintain a common concentration across treatments [21](Table S1). The experiments were conducted in randomized complete blocks (RCBD), with three replications from March 15 to April 28, 2017, and 30 healthy seeds of each line were used for each treatment.

The seeds of each line were sterilized for $15-20$ minutes in a $10 \% \mathrm{H}_{2} \mathrm{O}_{2}$ solution. After rinsing $5-6$ times in sterilized water, seeds were placed on moisturized germination paper in glass Petri dishes, crease-side down and left for $36 \mathrm{~h}$ in darkness for initial germination. After the early appearance of germination seeds were transferred to sand ( $2 \mathrm{~mm}$ diameter) box in a dark environment for seedling growth at $24^{\circ} \mathrm{C}$ 
for $72 \mathrm{~h}$. The sand box was kept under a constant environment room (12-h photo-period: $16^{\circ} \mathrm{C}$ day and $13^{\circ} \mathrm{C}$ night, light intensity at $400 \mu \mathrm{mol} \mathrm{m} \mathrm{m}^{-2} \mathrm{~s}^{-1}$ PAR while relative humidity at $70 \%$ ) where subsequent phenotyping was to be conducted for their growth. Three uniformly sprouted seeds with $\sim 5 \mathrm{~mm}$ in roots length were transferred to holes in trays (the seedling was holed with a sponge), and were placed on plastic tanks $(660 \times 480 \times 280 \mathrm{~mm})$ containing $20 \mathrm{~L}$ of nutrient solution; the solution was renewed every three days. After 10 days (two-leaf stage) plants were harvested and placed in $30 \%$ ethanol prior to imaging.

\section{Trait measurements}

Five root system architecture (RSA) traits, viz. root length (RL), root volume (RV), root diameter (RD), root tip number (RTN), root surface area (ROSA) were captured through images using a scanner (Perfection V700/V750 2.80A; Epson, China). Images were analyzed by using the software RootNav V1.7.5 which was operationally semi-automated [22]. Root biomass-related traits (RBT), including shoot dry weight (SDW) and root dry weight (RDW) were measured after oven-drying for $72 \mathrm{~h}$ at $70^{\circ} \mathrm{C}$ (mg/plant). Total dry weight (TDW) was estimated as the sum of SDW and RDW, and ratio of root to shoot dry weight (RRS) was measured as ratio between RDW and SDW.

\section{SNP genotyping and QTL analysis}

The DH lines and parent cultivars were genotyped through Wheat 660K SNP array synthesized by Affymetrix and commercially available from Capital Bio Corporation (Beijing, China; http://www.capitalbio.com). Genetic map was contacted by Wang et al. [23] from our lab. Briefly, markers with no polymorphisms between parents, severely distorted segregations, and missing rate greater than $20 \%$ were removed in the subsequent linkage analysis. Finally, 10,242 markers each representing a bin site were selected to construct the linkage map of Yangmai 16/Zhongmai 895 population, which comprised 25 linkage groups, covering all 21 wheat chromosomes. Among them, chromosomes 1B, 2B, $4 \mathrm{~A}$ and $7 \mathrm{~A}$ consisted of two linkage groups, and the remaining chromosomes were with only one linkage group. Inclusive composite-interval mapping was used for QTL analysis in IciMappingV4.1 software [24], and averaged data from the three replicates were used for QTL detection. The SNP genotypes of Yangmai 16 were defined as A, and those of Zhongmai 895 as B. Alleles from Yangmai 16 reduced trait values when the additive effects were negative. Kosambi mapping approach was used to convert recombinant frequencies into distance map [25]. Locations of QTLs for the root traits were detected by inclusive composite interval mapping-additive (ICIM-ADD) by using same software as for the QTL analysis. The threshold for significant QTL of each trait was demarcated by 1000-permutations at $P=0.05$ [26], and minimum LOD score at 2.5 with walking speed at $1.0 \mathrm{cM}$. Joint QTL analysis for closely linked pleiotropic QTL was performed using the general linear model scripted in Im package of R software. Phenotypic variance explained by each QTL was calculated as demonstrated in Li et al. [27].

Statistical analysis 
Pearson's correlations analysis among the traits were estimated using averaged data from each replicate. Significance of variances among $\mathrm{DH}$ lines, treatments and interactions between genotypes and treatments $(\mathrm{G} \times \mathrm{T})$ was calculated using following mixed linear model and considered significant at $P$ $<0.05$.

$Y=X \beta+Z \mu+\mathrm{ge}+\varepsilon(1)$

where $Y$ is demonstrated as the response from fixed $(\beta)$ and random $(\mu)$ effects with random error $(\varepsilon)$, ge is the genotype $\times$ environment effect while $X$ and $Z$ illustrate fixed and random effects, respectively. Broad sense heritabilities for all traits were estimated using genotypes as a random effect following [28].

$h^{2}=\sigma_{g}^{2 /}\left(\sigma_{g}^{2}+\sigma_{g t}^{2} / r+\sigma_{\varepsilon}^{2 / r t}\right)(2)$

where $\sigma_{g}{ }^{2}, \sigma_{g t}{ }^{2}$ and $\sigma_{\varepsilon}{ }^{2}$ represent genotype, genotype ( $\mathrm{DH}$ line) $\times \mathrm{P}$ treatment interaction and error variances, respectively, while $t$ is indicated $P$ treatments and $r$ is replicates. The $R$ package was used for all statistical analyses [29].

\section{Results}

Phenotypic variation and correlations among traits under $P$ treatments

Data for all nine traits were normally distributed across the three P treatments (Fig. S1). Under low P condition, Zhongmai 895 showed higher RL, RV, ROSA, RDW, TDW and RRS, but lower RD, RTN, and SDW than Yangmai 16. Except for RD and RRS in the high P treatment, Yangmai 16 had higher RL, RV, RTN, ROSA, SDW, RDW and TDW than Zhongmai 895 (Fig. S2).

Phenotypic variances among the $\mathrm{DH}$ lines were significant $(P<0.0001)$. Transgressive segregations across $P$ treatments were observed for most of traits (Fig. S2; Table 1). The average values of the DH lines for RL, RV, RTN, and RRS were higher than parents in the low $\mathrm{P}$ treatment, indicating positive effects for root vigor from both parents (Fig. 1खS2). Broad-sense heritabilities of nine traits were ranged from 0.76 to 0.91 (Table 1 ).

RSA and RBT traits were correlated significantly ( $r=0.59$ to 0.98 at all three P levels). However, RD was negatively correlated with RL, RTN and ROSA ( $r=-0.23$ to -0.69$)$ (Fig. 2).

\section{QTLs for root system architecture traits}

Nineteen QTLs from the three P treatments were identified for RSA traits RL, RV, RTN and ROSA on chromosomes 1BL, 2BL, 2DL, 3DL, 4BS, 6AL, 6BL (2), 6DS, 7AS (5), 7AL (3) and 7BL (2) (Table 2). Among them 6 QTLs were detected for the zero control, and 7 in the low and 6 in the high P treatments with the phenotypic variances explained of 45.1, 48.0, 53.7\%, respectively. Seventeen QTLs on chromosomes 2BL, 2DL, 3DL (2), 4BS (3), 6AL, 6BL (3), 6DS (2), 7AS (2), 7AL (2) conferred positive additive effects contributed by Zhongmai 895 (Table 2). In the low P treatment, three QTLs were identified for each of RL 
and RTN, explaining $20.7 \%$ and $21.7 \%$ of phenotypic variances, respectively. RL and RTN were co-located on chromosomes 6BL, 7AL and 7BL. In addition, Zhongmai 895 possessed positive alleles on 6BL and 7AL for increased phenotypic values (Table 2).

\section{QTLs for root biomass-related traits}

Fifteen QTLs for RBT traits were identified on chromosomes 3AS (3), 3DL, 4BS (5), 4DS (4), 6BL and 6DS (Table 2). Two, 4 and 9 QTLs were identified in the zero, low and high $P$ treatments, and explained 34.0, 42.8 and $67.6 \%$ of the phenotypic variances, respectively. A stable QTL (QRDW.caas-4BS) was identified under both low and high $\mathrm{P}$ conditions, explaining 8.1 to $17.7 \%$ of the phenotypic variances for RDW. A pleiotropic QTL on chromosome 4DS in interval $A X-109816583$ - $A X-109478820$ (16.64 - $30.66 \mathrm{Mb}$ ) detected in the low P treatment for RDW co-located with 3 QTLs in all three P treatments for RRS explained 7.1 to $20.4 \%$ of the phenotypic variances (Table 2 ).

\section{QTL clusters}

Fifteen QTLs in seven clusters (C1 to C7) for different traits in all three P treatments were identified in the same or close marker intervals (Fig. 3). QTL clusters were located on 3DL, 4BS, 4DS, 6BL, 7AS, 7AL and 7BS chromosomes, respectively. Of these, $\mathrm{C} 1$ was on 3DL for SDW and RTN under high $\mathrm{P}$ treatment. $\mathrm{C} 2$ for RV and RRS on 4BS was detected in a $0.31 \mathrm{Mb}$ interval between SNPs $A X-109491270(21.79 \mathrm{Mb})$ and $A X-108815849$ (21.42 Mb). C3 on 4DS comprised QTLs for RDW and RRS. QTL in clusters on 4B $(16.64-30.66 \mathrm{Mb})$ and $4 \mathrm{D}(32.42-37.86 \mathrm{Mb})$ were at or very near to reduced plant height gene loci Rht$B 1(30.8 \mathrm{Mb})$ and Rht-D1 (18.9Mb), respectively. C4, C6 and C7 involved the same traits (RL and RTN) on chromosomes 6BL, 7AL and 7BL. Joint QTL analysis reveal that, QTLs presented in four clusters. i.e. C3 (RDW and RRS), C4 (RL and RTN), C5 (RL, RTN, ROSA) and C7 (RL and RTN) were identified as pleiotropic QTL under different $P$ levels (Fig. 3). C5 on 7AS (AX-109955164-AX-109445593) affected RL, RTN and ROSA, with the favorable allele contributed by Zhongmai 895 (Fig. 3).

\section{Discussion}

\section{Evaluation of traits related to root system architecture and root biomass}

Using a hydroponic-based phenotyping pipeline the root biomass and root system architecture-related traits of a DH population were measured in a cost-effective and rapid manner. Previous studies found strong correlations between such laboratory-based experiments and field data [30,31]. Most of the traits were higher under the high $\mathrm{P}$ treatment compared with zero and low $\mathrm{P}$ treatments. High heritabilities and significant genetic variances for root system architecture and biomass traits indicated that these traits could be used as primary selection criteria for enhancement of $P$ uptake $[12,13]$. The present results corroborated earlier findings for genetic variances of root-related traits under different treatments $[6,32$, 33].

Correlation between root traits at variable P levels 
$P$ deprivation restricts the growth of main roots while increasing the lateral roots elongation, numbers of root hairs [34] (Gu et al. 2016) and leads to higher ratio of root to shoot that change the root architecture [10] (Ayalew et al. 2015). In this study, RSA traits RL, RV, RTN and ROSA, and RBT traits RDW and RRS showed higher growth and highly positive correlation between each other at the low P level (Fig. 1, 2). This trend was contributed by Zhongmai 895 , which also had longer roots, high root tip number and high RRS in the low $\mathrm{P}$ treatment. High accumulation of SDW under high $\mathrm{P}$ conditions and greater RRS under low P were observed in Yangmai 16 (Fig. S2). SDW and RRS were negatively correlated across treatments (Fig. 2), although previous work found that low $P$ led to reduced root biomass and had a significant impact on root system architecture traits $[4,13,35]$.

A significantly negative correlation ranging from -0.23 to -0.69 for RD and RL, RTN and ROSA in all three $P$ treatments indicated a negative association of RD with $\mathrm{P}$ uptake across all DH lines (Fig. 2). A similar trend also observed for Zhongmai 895 under high P treatment (Fig. S2).

\section{QTLs under phosphorus treatments}

Significant variances among genotypes allowed us to explore genomic regions associated with the observed traits [4]. Several QTLs were identified previously using hydroponic culture, which showed a vital role in nutrient uptake and a positive correlation with yield-related morphological traits. Here we identified 34 QTLs on 1B, 2B, 2D, 3A, 3D, 4B, 4D, 6A, 6B, 6D, 7A and 7B chromosomes across the $P$ treatments (Table 2). Some QTLs were already reported for root system architecture and root biomass-related traits $[12,36]$. New loci closely linked with $A X-109273188(16.63 \mathrm{Mb})$ on 3AS, $A X-94471535(704.88 \mathrm{Mb})$ on 6BL, $A X-109109966788(725.54 \mathrm{Mb})$ on 7AL and $A X-109289805(705.84 \mathrm{Mb})$ on 7BL showed significant influence for root growth.

The genetic diversity for root vigor under low $\mathrm{P}$ conditions that could potentially improve $\mathrm{P}$ acquisition efficiency, and described some QTLs for RBT under low P conditions [11]. We detected QTLs on 2BL, 4BS, 4DS, 6BL, 7AL and 7BL showing high phenotypic variances for RTN, RRS and RL under low P. (Table 2). Previously, Su et al. $[9,35]$ evaluated two DH populations in pot and field experiments and reported that common QTL on chromosome 4B associated with shoot biomass and tiller number were potentially important for P efficiency. Here, QTL in same genomic region of the 4B chromosome were detected for RDW and RRS (Table 2). Interactions between root-related growth traits and $R$ ht genes were reported as important in early vigor and nutrient uptake $[11,37,38,39]$. These demonstrated contrasting impact of $R h t$ genes on seedling traits under different nutrient conditions. In this study, two important QTLs mapped on 4B (16.64-30.66 Mb) and 4D (32.42-37.86 Mb) in low Pcorresponding to Rht-B1b (30.9Mb) and Rht$D 1 b(18.9 \mathrm{Mb})$, respectively, had negative impact on RRS for both parents and $\mathrm{DH}$ population in contrast with a previous report [38].

QTLs for RTN, RL and SDW were identified on 2DL and 6BL under low P condition. These QTLs had been also demonstrated for root related traits under $P$ sufficiency conditions on $2 \mathrm{D}$ and $6 \mathrm{~B}$ in pot trials [35] and hydroponic culture [4]. Five QTLs on chromosome 7AS explained higher phenotypic variances of 4.6 to $11.9 \%$ for RL, RTN and ROSA. Four of these QTLs detected under high P conditions negatively influenced 
root elongation, RTN and ROSA and the remaining QTLs on 7AL affecting RTN had a role in increasing RTN in low P condition (Table 2). RL, RTN and ROSA are important traits for nutrient uptake and early seedling vigor. Closely linked QTLs QRL.caas-7BL and QRTN.caas-7BL near AX-109289805 (705.84 $\mathrm{Mb}$ ) could be important for early vigor under $P$ deficient conditions, whereas their strong relationship with kernel number per spike was earlier demonstrated by Zhang et al. [40]. SNPs linked with QTLs for these traits on 7A and 7B stably detected in different nutrient conditions might be of great value for wheat breeding.

\section{QTL clusters}

Previously high-density physical mapping results demonstrated about $85 \%$ of gene expression and also has many cluster distributions in wheat genome that covered 5 to $10 \%$ of chromosome regions [41]. In wheat, many QTL clusters were reported in several studies [42-46]. In the present study, seven clusters were identified in three different P treatments (Fig. 3). Clusters C3, C4, C5 and C7 had pleiotropic QTLs for root system architecture and biomass-related traits under different $P$ treatments, whereas QTLs in other clusters contained closely linked multiple genes without pleiotropic effects. Clusters $\mathrm{C} 2$ and $\mathrm{C} 3$ on chromosomes 4BS and 4DS were linked with reduced plant height genes. Cluster C4 on 6BL had QTLs controlling RL and RTN and previously were reported for thousand grain weight [13]. Cluster C5 on chromosome 7AS (AX-109955164 - AX-109445593) affecting RL, RTN and ROSA was identified for the first time in the present study and SNP linked with this cluster can be used for future wheat improvement. Whereas cluster C7 on chromosome 7BL containing QTLs for RL and RTN was also reported for RL and SDW under P-deficient condition in previous studies [4, 13](Fig. 3). The SNPs tightly linked to QTLs or QTL clusters identified in the present study can be converted to KASP assays and effectively used for MAS to improve nutrient-use efficiency in wheat breeding.

\section{Conclusions}

Thirty-four QTLs with significant phenotypic variations for root system architecture and biomass-related traits were identified using high-density genetic map constructed from 660k SNP array and cost effective hydroponic-based phenotyping pipeline. Four QTLs on chromosomes 6BL (2) and 7AL (2) identified in low $\mathrm{P}$ treatment showed positive additive effects contributed by Zhongmai 895, indicating that Zhongmai 895 could be used as parent for P-deficient breeding. A stable QTL QRRS.caas-4DS (16.64-30.66 $\mathrm{Mb}$ )was also detected across the three $\mathrm{P}$ levels, accounted for 8.4 to $20.4 \%$ of the phenotypic variances, which could be used for speedy selection of genotypes for P-uptake. Among the seven QTLs clusters, C5 identified on chromosome 7AS (AX-109955164-AX-109445593) affecting RL, RTN and ROSA could be vital for improving the nutrient use efficiency of roots. The identified chromosome regions, particularly the QTL clusters could be used for molecular marker-assisted selection in future breeding.

\section{Abbreviations}


RSA: Root system architecture;RBT: Root biomass-related traits;RL: Root length;RV: Root volume; RD: Root diameter;RTN: Root tip number;ROSA: Root surface area; SDW: Shoot dry weight; RDW: Root dry weight; TDW: Total dry weight; RRS: Ratio of root to shoot dry weight; PUE: Phosphorus use efficiency; DH: Double haploid; QTLs: Quantitative trait loci; LOD: Log-of-odds

\section{Declarations}

Ethics approval and consent to participate

Not applicable.

Consent to publish

Not applicable.

Availability of data and materials

The datasets used for this article are available (included in Additional files).

Competing interests

The authors declare that they have no competing interests.

\section{Funding}

This research was supported by National Natural Science Foundation of China (31671691), National Key Project (2016YFD0101804, 2016YFD0101802), and National Key Technology R\&D Program of China (2014BAD01B05). The founding played an important role in the data collecting, analyzing and manuscript writing.

\section{Authors' contributions}

Performed the experiments: MY and CW. Designed and conceived the experiments: SS, XX, YX, ZH. Statistical data analysis: MY, MAH and FL. Wrote the paper: MY and MAH. All authors read and approved the final version of the manuscript.

\section{Acknowledgements}

We are thankful to Dr. R. A. Mclntosh at the Plant Breeding Institute, University of Sydney, and Awais Rasheed from CIMMYT China office, for reviewing this manuscript.

\section{References}

1. Malhi SS, Vera CL, Brandt SA. Seed yield potential of five wheat species/cultivars without and with phosphorus fertilizer application on a p-deficient soil in northeastern Saskatchewan. Agri Sci. 
2015;6(02):224-231.

2. James RA, Weligama C, Verbyla K, Ryan PR, Rebetzke GJ, Rattey A, Richardson AE, Delhaize E. Rhizosheaths on wheat grown in acid soils: phosphorus acquisition efficiency and genetic control. $J$ Exp Bot. 2016;67:3709-3718.

3. Wang W, Ding GD, White PJ, Wang XH, Jin KM, Xu FS, Shi L. Mapping and cloning of quantitative trait loci for phosphorus efficiency in crops: opportunities and challenges. Plant Soil. 2018:1-22.

4. Guo Y, Kong FM, Xu YF, Zhao Y, Liang X, Wang YY, An DG, Li SS. QTL mapping for seedling traits in wheat grown under varying concentrations of N, P and K nutrients. Theor Appl Genet. 2012;124:851865.

5. Carvalho P, Azam-Ali S, Foulkes MJ. Quantifying relationships between rooting traits and water uptake under drought in Mediterranean barley and durum wheat. J Integr Plant Biol. 2014;56:455469.

6. Liu Z, Gao K, Shan S, Gu R, Wang Z, Craft EJ, Mi G, Yuan L, Chen F. Comparative analysis of root traits and the associated QTL for maize seedlings grown in paper roll, hydroponics and vermiculite culture system. Front Plant Sci. 2017;8:436.

7. Clárk RB. Plant genotype differences in the uptake, translocation, accumulation, and use of mineral elements required for plant growth. Plant Soil. 1983;72:175-196.

8. Habash DZ, Bernard S, Schondelmaier J, Weyen J, Quarrie SA. The genetics of nitrogen use in hexaploid wheat: N utilisation, development and yield. Theor Appl Genet. 2007;114:403-419.

9. Su JY, Xiao YM, Li M, Liu QY, Li B, Tong YP, Jia JJ, Li ZS. Mapping QTL for phosphorus-deficiency tolerance at wheat seedling stage. Plant Soil. 2006;281:25-36.

10. Ayalew H, Ma X, Yan G. Screening wheat (Triticum spp.) genotypes for root length under contrasting water regimes: potential sources of variability for drought resistance breeding. J Agron Crop Sci. 2015;201:189-194.

11. Ryan PR, Liao M, Delhaize E, Rebetzke GJ, Weligama C, Spielmeyer W, James RA. Early vigour improves phosphate uptake in wheat. J Exp Bot. 2015;66:7089-7100.

12. Yuan Y, Gao M, Zhang M, Zheng H, Zhou X, Guo Y, Zhao Y, Kong F, Li S. QTL mapping for phosphorus efficiency and morphological traits at seedling and maturity stages in wheat. Front Plant Sci. 2017;8:614.

13. Zhang H, Wang HG. QTL mapping for traits related to P-deficient tolerance using three related RIL populations in wheat. Euphytica. 2015;203:505-520.

14. Bovill WD, Huang CY, McDonald GK. Genetic approaches to enhancing phosphorus-use efficiency (PUE) in crops: challenges and directions. Crop Pasture Sci. 2013;64:179-198.

15. Gong X, McDonald GK. QTL mapping of root traits in phosphorus-deficient soils reveals important genomic regions for improving NDVI and grain yield in barley. Theor Appl Genet. 2017;130:18851902. 
16. Atkinson JA, Wingen LU, Griffiths M, Pound MP, Gaju O, Foulkes MJ, Le Gouis J, Griffiths S, Bennett MJ, King J, Wells DM. Phenotyping pipeline reveals major seedling root growth QTL in hexaploid wheat. J Exp Bot. 2015;66:2283-2292.

17. Kabir MR, Liu G, Guan P, Wang F, Khan AA, Ni Z, Yao Y, Hu Z, Xin M, Peng H, Sun Q. Mapping QTL associated with root traits using two different populations in wheat (Triticum aestivum L.). Euphytica. 2015;206:175-190.

18. Cui F, Zhang N, Fan X, Zhang W, Zhao C, Yang L, Pan R, Chen M, Han J, Zhao X, Ji J, Tong Y, Zhang H, Jia J, Zhao G, Li M. Utilization of a Wheat 660K SNP array-derived high-density genetic map for highresolution mapping of a major QTL for kernel number. Sci Rep. 2017;7:3788.

19. Jin H, Wen W, Liu J, Zhai S, Zhang Y, Yan J, Liu Z, Xia X, He Z. Genome-wide QTL mapping for wheat processing quality parameters in a Gaocheng 8901/Zhoumai 16 recombinant inbred line population. Front Plant Sci. 2016;7:1032.

20. Hoagland DR, Arnon DI. The water culture method for growing plants without soil. Calif Agric Exp Stn Bull. 1938;347:36-39.

21. Li F, Xiao Y, Jing S, Xia X, Chen X, Wang H, He Z. Genetic analysis of nitrogen and phosphorus utilization efficiency related traits at seedling stage of Jing 411 and its derivatives. J Triticeae Crops. 2015;35:737-746 (in Chinese with English abstract).

22. Pound MP, French AP, Atkinson J, Wells DM, Bennett MJ, Pridmore TP. RootNav: Navigating images of complex root architectures. Plant Physiol. 2013;162:1802-1814.

23. Wang JP. Genetic analysis and functional marker development of key traits in wheat. Dissertation, Shandong Agricultural University, 2017(in chinese).

24. Meng L, Li H, Zhang L, Wang J. QTL IciMapping: Integrated software for genetic linkage map construction and quantitative trait locus mapping in biparental populations. Crop J. 2015;3:269283.

25. Kosambi DD. The estimation of map distances from recombination values. Annu Eugen. 1944;12:172-175.

26. Churchill GA, Doerge RW. Empirical threshold values for quantitative trait mapping. Genetics. 1994;138:963-971.

27. Li H, Bradbury P, Ersoz E, Buckler ES, Wang J. Joint QTL linkage mapping for multiple-cross mating design sharing one common parent. PLoS One. 2011;6:e17573.

28. Sehgal D, Skot L, Singh R, Srivastava RK, Das SP, Taunk J, Sharma PC, Pal R, Raj B, Hash CT, Yadav RS. Exploring potential of pearl millet germplasm association panel for association mapping of drought tolerance traits. PLoS One. 2015;10:e0122165.

29. R Core Team. R: A language and environment for statistical computing. R Foundation for Statistical Computing, Vienna, Austria. 2013.

30. Ren Y, Qian Y, Xu Y, Zou C, Liu D, Zhao X, Zhang A, Tong Y. Characterization of QTL for root traits of wheat grown under different nitrogen and phosphorus supply levels. Front Plant Sci. 2017;8:2096. 
31. Xie Q, Fernando KM, Mayes S, Sparkes DL. Identifying seedling root architectural traits associated with yield and yield components in wheat. Ann Bot. 2017;119:1115-1129.

32. Ao J, Fu J, Tian J, Yan X, Liao H. Genetic variability for root morpho-architecture traits and root growth dynamics as related to phosphorus efficiency in soybean. Funct Plant Biol. 2010;37:304312.

33. Bayuelo-Jiménez JS, Gallardo-Valdéz M, Pérez-Decelis VA, Magdaleno-Armas L, Ochoa I, Lynch JP. Genotypic variances for root traits of maize (Zea mays L.) from the Purhepecha Plateau under contrasting phosphorus availability. Field Crops Res. 2011;121:350-362.

34. Gu R, Chen F, Long L, Cai H, Liu Z, Yang J, Wang L, Li H, Li J, Liu W, Mi G, Zhang F, Yuan L. Enhancing phosphorus uptake efficiency through QTL-based selection for root system architecture in maize. J Genet Genomics. 2016;43:663-672.

35. Su JY, Zheng Q, Li HW, Li B, Jing RL, Tong YP, Li ZS. Detection of QTL for phosphorus use efficiency in relation to agronomic performance of wheat grown under phosphorus sufficient and limited conditions. Plant Sci. 2009;176:824-836.

36. Sun JJ, Guo Y, Zhang GZ, Gao MG, Zhang GH, Kong FM, Zhao Y, Li SS. QTL mapping for seedling traits under different nitrogen forms in wheat. Euphytica. 2013;191:317-331.

37. Wojciechowski T, Gooding MJ, Ramsay L, Gregory PJ. The effects of dwarfing genes on seedling root growth of wheat. J Exp Bot. 2009;60:2565-2573.

38. Bai C, Liang Y, Hawkesford MJ. Identification of QTL associated with seedling root traits and their correlation with plant height in wheat. J Exp Bot. 2013;64:1745-1753

39. Narayanan S, Vara Prasad PV. Characterization of a spring wheat association mapping panel for root traits. Agron J. 2014;106:1593-1604.

40. Zhang H, Chen J, Li R, Deng Z, Zhang K, Liu B, Tian J. Conditional QTL mapping of three yield components in common wheat (Triticum aestivum L.). Crop J. 2016;4:220-228.

41. Cui F, Ding A, Li J, Zhao C, Li X, Feng D, Wang X, Wang L, Gao J, Wang H. Wheat kernel dimensions: how do they contribute to kernel weight at an individual QTL level? J Genet. 2011;90:409-425.

42. Quarrie SA, Pekic Quarrie S, Radosevic R, Rancic D, Kaminska A, Barnes JD, Leverington M, Ceoloni C, Dodig D. Dissecting a wheat QTL for yield present in a range of environments: from the QTL to candidate genes. J Exp Bot. 2006;57:2627-2637.

43. Crossa J, Burgueño J, Dreisigacker S, Vargas M, Herrera-Foessel SA, Lillemo M, Singh RP, Trethowan R, Warburton M, Franco J, Reynolds M, Crouch JH, Ortiz R. Association analysis of historical bread wheat germplasm using additive genetic covariance of relatives and population structure. Genetics. 2007;177:1889-1913.

44. Kong FM, Guo Y, Liang X, Wu CH, Wang YY, Zhao Y, Li SS. Potassium (K) effects and QTL mapping for K efficiency traits at seedling and adult stages in wheat. Plant Soil. 2013;373:877-892.

45. Zhao Y, Li XY, Zhang SH, Wang J, Yang XF, Tian JC, Hai Y, Yang XJ. Mapping QTL for potassiumdeficiency tolerance at the seedling stage in wheat (Triticum aestivum L.). Euphytica. 2014;198:185198.

Page $12 / 19$ 
46. Gong X, Wheeler R, Bovill WD, McDonald GK. QTL mapping of grain yield and phosphorus efficiency in barley in a Mediterranean-like environment. Theor Appl Genet. 2016;129:1657-1672.

\section{Tables}

Table 1 Phenotypic variances for three $\mathrm{P}$ treatments in DH lines

\begin{tabular}{|c|c|c|c|c|c|c|c|c|}
\hline Trait & P Level & Min & Max & Mean+SD & $h^{2}$ & $\begin{array}{c}\mathrm{G} \\
\text { F.value }\end{array}$ & $\begin{array}{c}\mathrm{T} \\
\text { F.value } \\
\end{array}$ & $\begin{array}{c}\text { GXT } \\
\text { F.value }\end{array}$ \\
\hline \multirow[t]{3}{*}{$\mathrm{RL}(\mathrm{cm})$} & $\mathrm{C}$ & 18.13 & 56.86 & $33.07 \pm 10.72$ & 0.90 & $2.89 * *$ & $706.75^{* *}$ & $1.39 * *$ \\
\hline & $\mathrm{L}$ & 22.21 & 98.83 & $60.51 \pm 21.07$ & 0.83 & $1.53^{* *}$ & & \\
\hline & $\mathrm{H}$ & 2.01 & 63.37 & $40.00 \pm 12$ & 0.77 & 1.09 & & \\
\hline \multirow[t]{3}{*}{$\operatorname{RV}\left(\mathrm{cm}^{3}\right)$} & $\mathrm{C}$ & 0.05 & 0.23 & $0.11 \pm 0.03$ & 0.86 & $2.06^{* *}$ & $1252.17 * *$ & $1.46^{* *}$ \\
\hline & $\mathrm{L}$ & 0.04 & 0.34 & $0.17 \pm 0.05$ & 0.81 & $1.45^{* *}$ & & \\
\hline & $\mathrm{H}$ & 0.08 & 0.42 & $0.22 \pm 0.008$ & 0.87 & $2.28 * *$ & & \\
\hline \multirow[t]{3}{*}{$\mathrm{RD}\left(\mathrm{cm}^{3}\right)$} & $\mathrm{C}$ & 0.16 & 0.35 & $0.21 \pm 0.03$ & 0.87 & $2.27 * *$ & $2274.54^{* *}$ & $1.43^{* *}$ \\
\hline & $\mathrm{L}$ & 0.14 & 0.28 & $0.19 \pm 0.02$ & 0.86 & $2.01 * *$ & & \\
\hline & $\mathrm{H}$ & 0.08 & 0.42 & $0.23 \pm 0.06$ & 0.88 & $2.54^{* *}$ & & \\
\hline \multirow[t]{3}{*}{ RTN } & C & 209 & 1822 & $716.25 \pm 246.7$ & 0.91 & $3.19 * *$ & $403.58 * *$ & $1.34^{* *}$ \\
\hline & $\mathrm{L}$ & 183 & 2477 & $1090.1 \pm 352.41$ & 0.84 & $1.76^{* *}$ & & \\
\hline & $\mathrm{H}$ & 184 & 1593 & $836.63 \pm 341.52$ & 0.82 & $1.56 * *$ & & \\
\hline \multirow[t]{3}{*}{$\operatorname{ROSA}\left(\mathrm{cm}^{3}\right)$} & $\mathrm{C}$ & 7.25 & 39.36 & $21.44 \pm 5.24$ & 0.88 & $2.45^{* *}$ & $700.51 * *$ & $1.36^{* *}$ \\
\hline & $\mathrm{L}$ & 7.48 & 66.38 & $36.07 \pm 10.12$ & 0.82 & $1.48^{* *}$ & & \\
\hline & $\mathrm{H}$ & 9.78 & 66.83 & $33.99 \pm 9.32$ & 0.83 & $1.68^{* *}$ & & \\
\hline \multirow[t]{3}{*}{ SDW (mg) } & $\mathrm{C}$ & 0.02 & 2.06 & $0.04 \pm 0.009$ & 0.76 & 1.04 & $46.72^{* *}$ & 1.01 \\
\hline & $\mathrm{L}$ & 0.02 & 0.09 & $0.05 \pm 0.01$ & 0.86 & $2.09 * *$ & & \\
\hline & $\mathrm{H}$ & 0.005 & 0.12 & $0.071 \pm 0.009$ & 0.88 & $2.54 * *$ & & \\
\hline \multirow[t]{3}{*}{ RDW (mg) } & $\mathrm{C}$ & 0.01 & 0.03 & $0.02 \pm 0.001$ & 0.89 & $2.69 * *$ & $451.45^{* *}$ & $1.41 * *$ \\
\hline & $\mathrm{L}$ & 0.01 & 0.04 & $0.02 \pm 0.001$ & 0.84 & $1.72^{* *}$ & & \\
\hline & $\mathrm{H}$ & 0.01 & 0.12 & $0.07 \pm 0.02$ & 0.89 & $2.67 * *$ & & \\
\hline \multirow[t]{3}{*}{ TDW (mg) } & $\mathrm{C}$ & 0.01 & 2.08 & $0.06 \pm 0.008$ & 0.76 & 1.05 & $65.22^{* *}$ & 1.03 \\
\hline & $\mathrm{L}$ & 0.01 & 0.12 & $0.07 \pm 0.02$ & 0.85 & $1.88^{* *}$ & & \\
\hline & $\mathrm{H}$ & 0.01 & 0.04 & $0.02 \pm 0.001$ & 0.88 & $2.42^{* *}$ & & \\
\hline \multirow[t]{3}{*}{ RRS } & C & 0.01 & 0.71 & $0.38 \pm 0.07$ & 0.91 & $3.34^{* *}$ & $180.38^{* *}$ & 1.03 \\
\hline & $\mathrm{L}$ & 0.18 & 1.04 & $0.39 \pm 0.07$ & 0.83 & $1.68^{* *}$ & & \\
\hline & $\mathrm{H}$ & 0.01 & 0.16 & $0.09 \pm 0.02$ & 0.88 & $2.50 * *$ & & \\
\hline
\end{tabular}

* Significant at $P<0.05, * *$ at $P<0.001$ and ${ }^{* * *}$ at $P<0.0001$

Abbr: C, zero control; L, low P treatment; H, high P treatment; RL, Root length; RV, Root volume; RD, Root diameter; RTN, Root tip number; ROSA, Root surface area; SDW, Shoot dry weight; RDW, Root dry weight; TDW, Total dry weight; RRS, Ratio of root to shoot dry weight

Table 2 QTLs for root system architecture and root biomass-related traits identified under three $\mathrm{P}$ treatments 


\begin{tabular}{|c|c|c|c|c|c|c|c|}
\hline Trait & Treatment & $\mathrm{QTL}^{\mathrm{a}}$ & Marker interval & $\begin{array}{l}\text { Physical interval }^{\mathrm{b}} \\
(\mathrm{Mb})\end{array}$ & $\mathrm{LOD}^{\mathrm{c}}$ & $\begin{array}{l}\text { PVE }^{d} \\
(\%)\end{array}$ & Add $^{\mathrm{e}}$ \\
\hline \multirow[t]{8}{*}{ RL } & $\mathrm{C}$ & QRL.caas- $1 B L$ & $\begin{array}{l}A X-10928674-A X- \\
94446430\end{array}$ & 671.33-688.38 & 3.13 & 5.56 & -18.89 \\
\hline & & $\begin{array}{l}\text { QRL.caas- } \\
7 A S .1\end{array}$ & $\begin{array}{l}A X-110432090-A X- \\
109345074\end{array}$ & $20.95-22.87$ & 3.51 & 6.38 & 19.78 \\
\hline & & $Q R L . c a a s-6 A L$ & $\begin{array}{l}A X-86165298-A X- \\
109431293\end{array}$ & 612.11-613.49 & 4.53 & 8.20 & 23.51 \\
\hline & & QRL.caas-7AL & $\begin{array}{l}A X-109966788-A X- \\
94819074\end{array}$ & 725.54-734.54 & 3.37 & 6.22 & 19.54 \\
\hline & $\mathrm{L}$ & QRL.caas-6BL & $\begin{array}{l}A X-109368729-A X- \\
95094583\end{array}$ & 699.99-711.76 & 4.90 & 8.61 & 45.43 \\
\hline & & QRL.caas-7BL & $\begin{array}{l}A X-95025477-A X- \\
109289805\end{array}$ & 700.83-705.84 & 3.74 & 6.57 & -38.04 \\
\hline & & QRL.caas-7AL & $\begin{array}{l}A X-109966788-A X- \\
94819074\end{array}$ & $725.54-734.54$ & 3.46 & 5.50 & 35.19 \\
\hline & $\mathrm{H}$ & $\begin{array}{l}\text { QRL.caas- } \\
7 A S .2\end{array}$ & $\begin{array}{l}A X-109955164-A X- \\
109445593\end{array}$ & $116.62-122.18$ & 4.72 & 11.89 & 30.81 \\
\hline \multirow[t]{3}{*}{ RV } & C & $Q R V . c a a s-6 D S$ & $\begin{array}{l}A X-10934618-A X- \\
94913665\end{array}$ & $18.67-25.66$ & 3.98 & 8.41 & 0.01 \\
\hline & & QRV.caas- $4 B S$ & $\begin{array}{l}A X-108815849-A X- \\
109491270\end{array}$ & $21.48-21.79$ & 4.90 & 10.32 & 0.01 \\
\hline & $\mathrm{H}$ & QRV.caas-7AS & $\begin{array}{l}A X-108763612-A X- \\
111263013\end{array}$ & $85.44-85.71$ & 3.49 & 8.82 & 0.01 \\
\hline \multirow[t]{7}{*}{ RTN } & $\mathrm{L}$ & $\begin{array}{l}\text { QRTN.caas- } \\
6 B L\end{array}$ & $\begin{array}{l}A X-109368729-A X- \\
95094583\end{array}$ & 699.99-711.76 & 4.21 & 7.08 & 73.56 \\
\hline & & $\begin{array}{l}\text { QRTN.caas- } \\
2 B L\end{array}$ & $\begin{array}{l}A X-86176885-A X- \\
94933613\end{array}$ & $647.32-647.71$ & 2.86 & 4.79 & 57.39 \\
\hline & & $\begin{array}{l}\text { QRTN.caas- } \\
7 B L\end{array}$ & $\begin{array}{l}A X-95025477-A X- \\
109289805\end{array}$ & 700.83-705.84 & 5.52 & 9.78 & -81.68 \\
\hline & & $\begin{array}{l}\text { QRTN.caas- } \\
7 A L\end{array}$ & $\begin{array}{l}A X-109966788-A X- \\
94819074\end{array}$ & 725.54-734.54 & 2.77 & 4.64 & 56.97 \\
\hline & $\mathrm{H}$ & $\begin{array}{l}\text { QRTN.caas- } \\
2 D L\end{array}$ & $\begin{array}{l}A X-94959623-A X- \\
94821426\end{array}$ & $79.54-82.22$ & 2.74 & 7.78 & 45.97 \\
\hline & & $\begin{array}{l}\text { QRTN.caas- } \\
7 A S\end{array}$ & $\begin{array}{l}A X-109955164-A X- \\
109445593\end{array}$ & 116.62-122.18 & 3.84 & 8.17 & 48.20 \\
\hline & & $\begin{array}{l}\text { QRTN.caas- } \\
3 D L\end{array}$ & $\begin{array}{l}A X-110036411-A X- \\
109917936\end{array}$ & $559.77-566.72$ & 2.99 & 6.21 & 42.26 \\
\hline
\end{tabular}




\begin{tabular}{|c|c|c|c|c|c|c|c|}
\hline ROSA & $\mathrm{H}$ & $\begin{array}{l}\text { QROSA.caas- } \\
\text { 7AS }\end{array}$ & $\begin{array}{l}A X-109955164-A X- \\
109445593\end{array}$ & $116.62-122.18$ & 4.43 & 10.80 & 2.24 \\
\hline \multirow[t]{4}{*}{ SDW } & $\mathrm{H}$ & $\begin{array}{l}\text { QSDW.caas- } \\
6 B L\end{array}$ & $\begin{array}{l}A X-109368729-A X- \\
95094583\end{array}$ & 699.99-711.76 & 3.44 & 6.80 & 0.00 \\
\hline & & $\begin{array}{l}\text { QSDW.caas- } \\
6 D S\end{array}$ & $\begin{array}{l}A X-109346183-A X- \\
94913665\end{array}$ & $18.67-25.66$ & 2.59 & 4.95 & 0.00 \\
\hline & & $\begin{array}{l}\text { QSDW.caas- } \\
3 A S\end{array}$ & $\begin{array}{l}A X-111507145-A X- \\
109273188\end{array}$ & $15.80-16.63$ & 3.01 & 5.99 & 0.00 \\
\hline & & $\begin{array}{l}\text { QSDW.caas- } \\
3 D L\end{array}$ & $\begin{array}{l}A X-110036411-A X- \\
109917936\end{array}$ & $559.77-566.72$ & 4.17 & 8.21 & 0.00 \\
\hline \multirow[t]{4}{*}{ RDW } & $\mathrm{L}$ & $\begin{array}{l}Q R D W . c a a s- \\
4 B S\end{array}$ & $\begin{array}{l}A X-111068079-A X- \\
111164540\end{array}$ & $32.42-37.86$ & 7.70 & 17.70 & 0.00 \\
\hline & & $\begin{array}{l}Q R D W . c a a s- \\
4 D S\end{array}$ & $\begin{array}{l}A X-109816583-A X- \\
109478820\end{array}$ & $16.64-30.66$ & 3.12 & 7.14 & 0.00 \\
\hline & $\mathrm{H}$ & $\begin{array}{l}\text { QRDW.caas- } \\
3 A S\end{array}$ & $\begin{array}{l}A X-111507145-A X- \\
109273188\end{array}$ & $15.80-16.63$ & 3.08 & 7.06 & 0.00 \\
\hline & & $\begin{array}{l}\text { QRDW.caas- } \\
4 B S\end{array}$ & $\begin{array}{l}A X-111068079-A X- \\
111164540\end{array}$ & $32.42-37.86$ & 3.59 & 8.12 & 0.00 \\
\hline TDW & $\mathrm{H}$ & $\begin{array}{l}\text { QTDW.caas- } \\
3 A S\end{array}$ & $\begin{array}{l}A X-111507145-A X- \\
109273188\end{array}$ & $15.80-16.63$ & 3.11 & 7.68 & 0.00 \\
\hline \multirow[t]{6}{*}{ RRS } & $\mathrm{C}$ & $\begin{array}{l}\text { QRRS.caas- } \\
4 B S .1\end{array}$ & $\begin{array}{l}A X-109494015-A X- \\
108991675\end{array}$ & $97.25-103.06$ & 6.78 & 13.59 & 0.01 \\
\hline & & $\begin{array}{l}\text { QRRS.caas- } \\
4 D S\end{array}$ & $\begin{array}{l}A X-109816583-A X- \\
109478820\end{array}$ & 16.64-30.66 & 10.43 & 20.41 & -0.01 \\
\hline & $\mathrm{L}$ & $\begin{array}{l}\text { QRRS.caas- } \\
4 B S .1\end{array}$ & $\begin{array}{l}A X-109494015-A X- \\
108991675\end{array}$ & $97.25-103.06$ & 2.94 & 6.25 & 0.01 \\
\hline & & $\begin{array}{l}\text { QRRS.caas- } \\
4 D S\end{array}$ & $\begin{array}{l}A X-109816583-A X- \\
109478820\end{array}$ & 16.64-30.66 & 5.55 & 11.71 & -0.01 \\
\hline & $\mathrm{H}$ & $\begin{array}{l}\text { QRRS.caas- } \\
4 B S .2\end{array}$ & $\begin{array}{l}A X-108815849-A X- \\
109491270\end{array}$ & $21.48-21.79$ & 4.33 & 9.35 & 0.01 \\
\hline & & $\begin{array}{l}\text { QRRS.caas- } \\
4 D S\end{array}$ & $\begin{array}{l}A X-109816583-A X- \\
109478820\end{array}$ & 16.64-30.66 & 3.85 & 8.30 & -0.01 \\
\hline
\end{tabular}

a Quantitative trait loci

b Physical positions of SNP markers based on wheat genome sequences from the International Wheat Genome Sequencing Consortium (IWGSC, http://www.wheatgenome.org/)

c LOD value of each QTL 
d Phenotypic variance explained by QTL

e A positive sign means the increased effect contributed by Zhongmai 895, whereas the negative effect was contributed by Yangmai 16

Abbr: C, control; L, low P treatment; H, high P treatment; RL, Root length; RV, Root volume; RD, Root diameter; RTN, Root tip number; ROSA, Root surface area; SDW, Shoot dry weight; RDW, Root dry weight; TDW, Total dry weight; RRS, Ratio of root to shoot dry weight.

$(\mathrm{Q}=\mathrm{QTL})+($ caas $=$ Chinese Academy of Agricultural Sciences $)+$ Chromosome=long arm $(\mathrm{L})$ or short arm $(\mathrm{S})$

Table 3: Joint QTL analysis results for pleiotropic QTL in clusters

\begin{tabular}{|c|c|c|c|c|c|c|c|}
\hline Cluster & Trait & $\begin{array}{l}\mathrm{P} \\
\text { Level }\end{array}$ & Chromosome & Marker interval & $\begin{array}{l}\text { Physical } \\
\text { interval }^{\mathrm{a}}(\mathrm{Mb})\end{array}$ & $\operatorname{Pr}(>|t|)^{b}$ & $\begin{array}{l}\text { Adjusted } \\
\mathrm{R}^{2}\end{array}$ \\
\hline \multirow[t]{2}{*}{$\mathrm{C} 1$} & SDW & $\mathrm{H}$ & $3 \mathrm{D}$ & $\begin{array}{l}A X-110036411-A X- \\
109917936\end{array}$ & $559.77-566.72$ & $0.02 *$ & 0.38 \\
\hline & RTN & $\mathrm{H}$ & & & & & \\
\hline \multirow[t]{2}{*}{$\mathrm{C} 2$} & $\mathrm{RV}$ & $\mathrm{C}$ & $4 B$ & $\begin{array}{l}A X-109491270-A X- \\
108815849\end{array}$ & $21.48-21.79$ & $0.05^{*}$ & 0.12 \\
\hline & RRS & $\mathrm{H}$ & & & & & \\
\hline \multirow[t]{2}{*}{$\mathrm{C} 3$} & RDW & $\mathrm{L}$ & $4 \mathrm{D}$ & $\begin{array}{l}A X-109816583-A X- \\
109478820\end{array}$ & $16.64-30.66$ & $0.15,0.18$ & $0.18,0.12$ \\
\hline & RRS & $\mathrm{C}, \mathrm{L}$ & & & & & \\
\hline \multirow[t]{2}{*}{$\mathrm{C} 4$} & $\mathrm{RL}$ & $\mathrm{L}$ & $6 B$ & $\begin{array}{l}A X-94471535-A X- \\
95094583\end{array}$ & 704.88-711.76 & 0.7 & 0.75 \\
\hline & RTN & $\mathrm{L}$ & & & & & \\
\hline \multirow[t]{3}{*}{ C5 } & $\mathrm{RL}$ & $\mathrm{H}$ & $7 \mathrm{~A}$ & $\begin{array}{l}A X-109955164-A X- \\
109445593\end{array}$ & $116.62-122.18$ & $\begin{array}{l}0.68,0.55, \\
0.71\end{array}$ & $\begin{array}{l}0.76,0.90, \\
0.74\end{array}$ \\
\hline & RTN & $\mathrm{H}$ & & & & & \\
\hline & ROSA & $\mathrm{H}$ & & & & & \\
\hline \multirow[t]{2}{*}{ C6 } & $\mathrm{RL}$ & $\mathrm{H}$ & $7 \mathrm{~A}$ & $\begin{array}{l}A X-109966788-A X- \\
94819074\end{array}$ & 725.54-134.54 & $0.037 *$ & 0.12 \\
\hline & RTN & $\mathrm{L}$ & & & & & \\
\hline \multirow[t]{2}{*}{ C7 } & $\mathrm{RL}$ & $\mathrm{L}$ & $7 \mathrm{~B}$ & $\begin{array}{l}A X-109289805-A X- \\
95025477\end{array}$ & 700.83-705.84 & 0.8 & 0.75 \\
\hline & RTN & $\mathrm{L}$ & & & & & \\
\hline
\end{tabular}

a Physical positions of SNP markers based on wheat genome sequences from the International Wheat Genome Sequencing Consortium (IWGSC, http://www.wheatgenome.org/)

b Significance of t-values at $P<0.05$

Abbr: C, control; L, low P treatment; H, high P treatment; RL, Root length; RV, Root volume; RD, Root diameter; RTN, Root tip number; ROSA, Root surface area; SDW, Shoot dry weight; RDW, Root dry weight; TDW, Total dry weight; RRS, Ratio of root to shoot dry weight. 
Figures

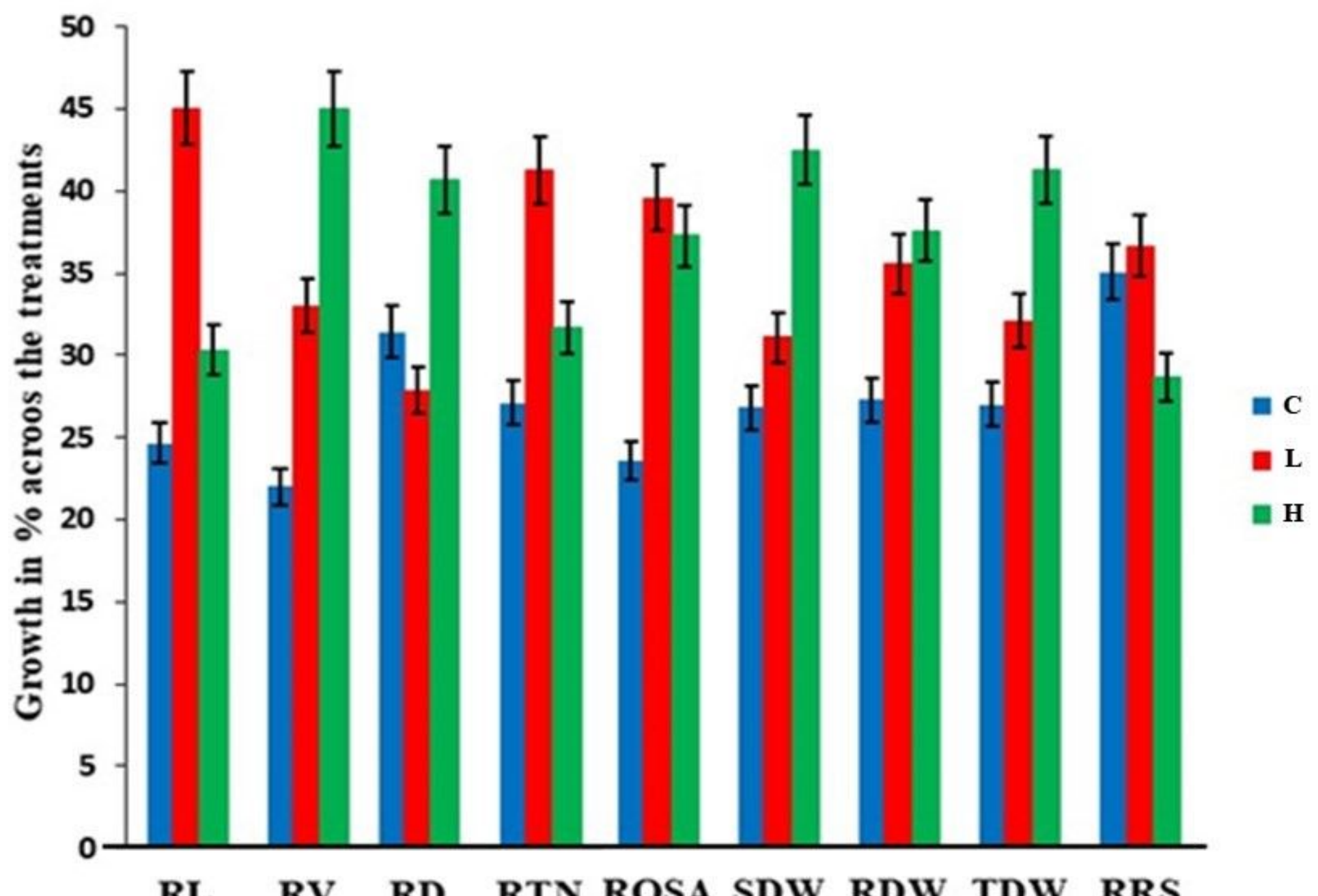

Figure 1

Effects of phosphorus treatment on traits in DH lines. Error bars represent standard deviation for each trait under $\mathrm{P}$ treatments. Abbr: $\mathrm{C}$, control; $\mathrm{L}$, low $\mathrm{P}$ treatment; $\mathrm{H}$, high $\mathrm{P}$ treatment; $\mathrm{RL}$, Root length; RV, Root volume; RD, Root diameter; RTN, Root tip number; ROSA, Root surface area; SDW, Shoot dry weight; RDW, Root dry weight; TDW, Total dry weight; RRS, Ratio of root to shoot dry weight. 

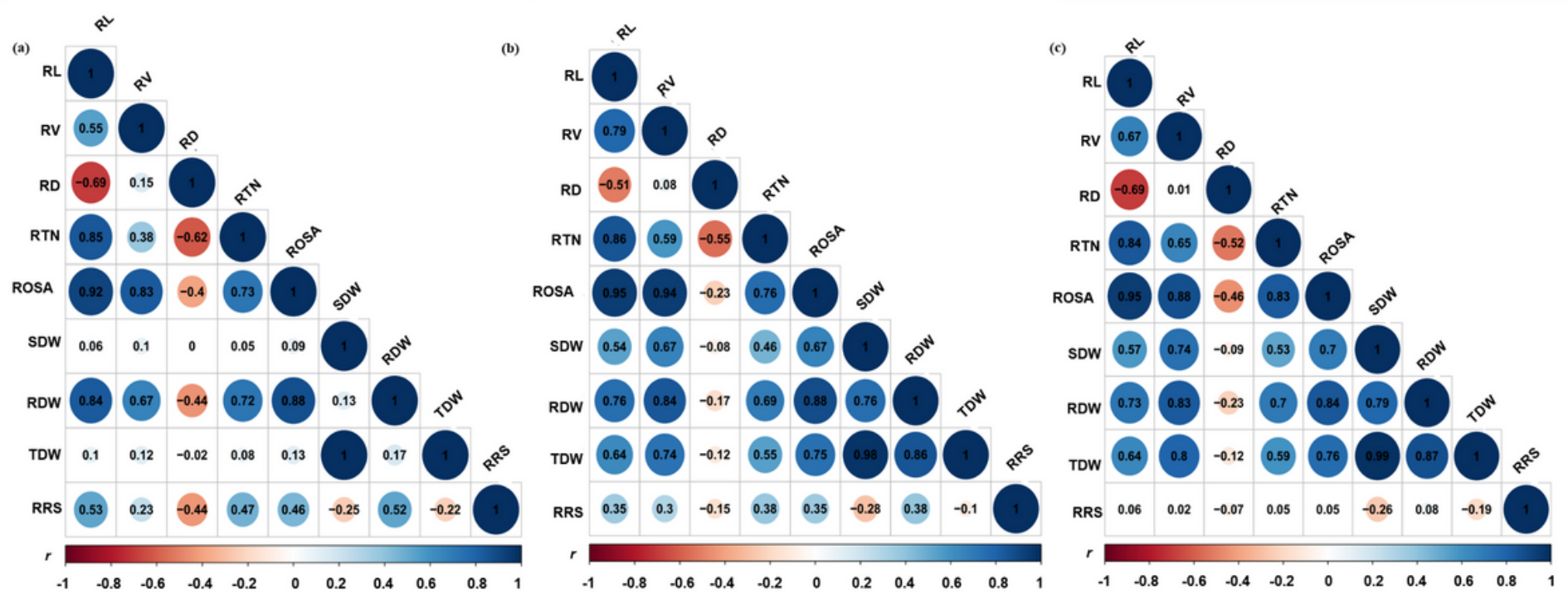

Figure 2

Correlation analysis among the traits under (a) control, (b) low and (c) high P treatment. Intensities of blue colour shows degrees of positive and red colour shows degrees of negative correlations; circle sizes indicate low to high significance. Abbr: RL, Root length; RV, Root volume; RD, Root diameter; RTN, Root tip number; ROSA, Root surface area; SDW, Shoot dry weight; RDW, Root dry weight; TDW, Total dry weight; RRS, Ratio of root to shoot dry weight. 

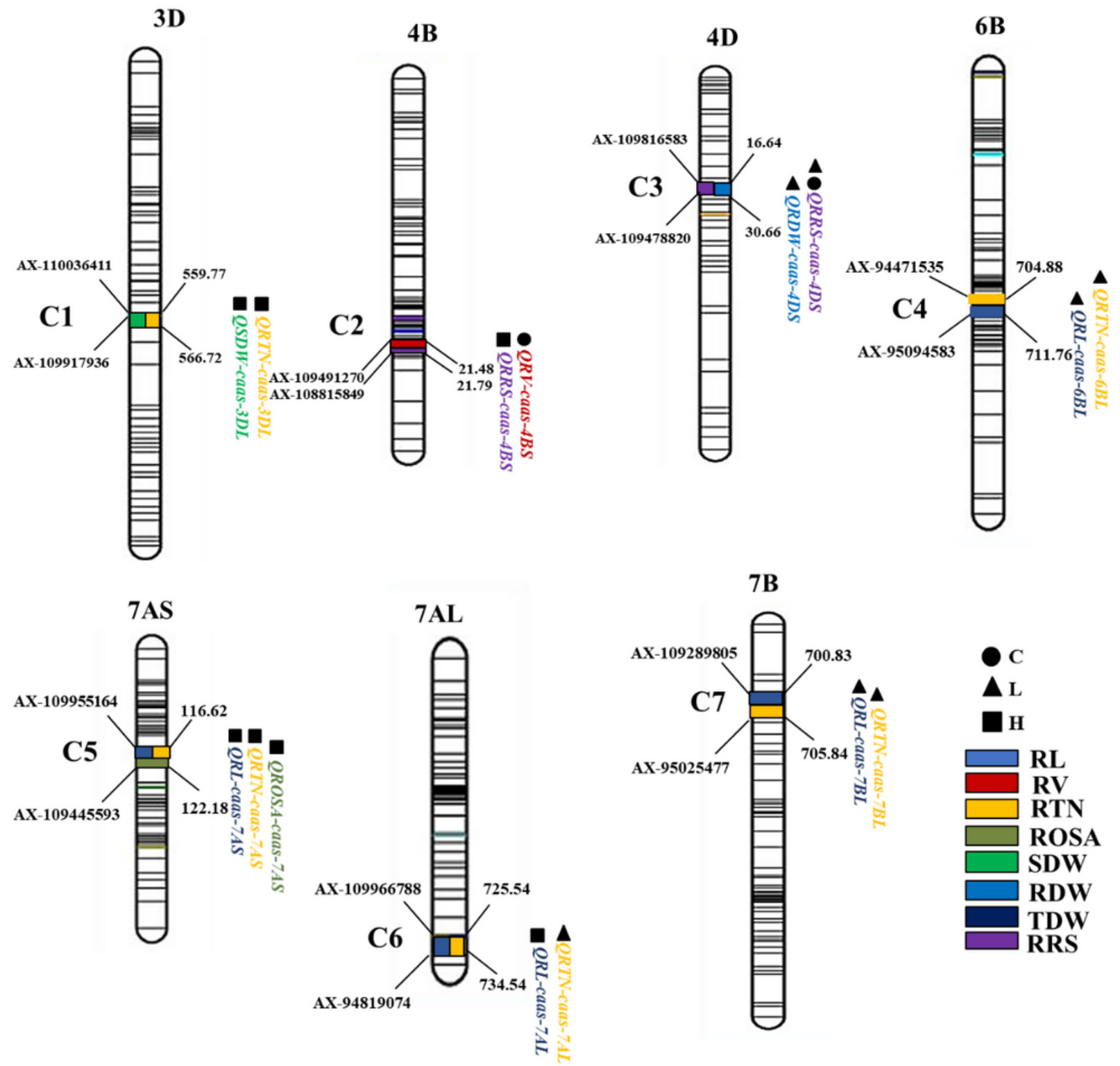

\section{Figure 3}

Seven QTL clusters containing pleiotropic loci with closely linked markers and physical positions. Abbr: C, control; L, low P treatment; $\mathrm{H}$, high P treatment; RL, Root length; RV, Root volume; RTN, Root tip number; ROSA, Root surface area; SDW, Shoot dry weight; RDW, Root dry weight; TDW, Total dry weight; RRS, Ratio of root to shoot dry weight.

\section{Supplementary Files}

This is a list of supplementary files associated with this preprint. Click to download.

- supplement1.docx 